Research Article

\title{
Generalized SOR-Like Iteration Method for Linear Complementarity Problem
}

\author{
Cui-Xia Li $(\mathbb{D})$ and Shi-Liang Wu \\ School of Mathematics, Yunnan Normal University, Kunming 650500, Yunnan, China \\ Correspondence should be addressed to Cui-Xia Li; lixiatk@126.com
}

Received 7 June 2020; Accepted 23 July 2020; Published 12 August 2020

Guest Editor: S. A. Edalatpanah

Copyright (C) 2020 Cui-Xia Li and Shi-Liang Wu. This is an open access article distributed under the Creative Commons Attribution License, which permits unrestricted use, distribution, and reproduction in any medium, provided the original work is properly cited.

In this paper, we present a generalized SOR-like iteration method to solve the non-Hermitian positive definite linear complementarity problem (LCP), which is obtained by reformulating equivalently the implicit fixed-point equation of the LCP as a two-by-two block nonlinear equation. The convergence properties of the generalized SOR-like iteration method are discussed under certain conditions. Numerical experiments show that the generalized SOR-like method is efficient, compared with the SORlike method and the modulus-based SOR method.

\section{Introduction}

The linear complementarity problem is to find $z \in \mathbb{R}^{n}$ such that

$$
w=M z+q \geq 0, \quad z \geq 0, \quad z^{T} w=0,
$$

where $M \in \mathbb{R}^{n \times n}$ is a given matrix and $q \in \mathbb{R}^{n}$ is a given vector, which is abbreviated as the $\operatorname{LCP}(q, M)$. Since the $\operatorname{LCP}(q, M)$ of form (1) often occurs in many actual problems of scientific computing and engineering applications, such as the linear and quadratic programming, the economies with institutional restrictions upon prices, the optimal stopping in Markov chain, and the free boundary problems, its numerical solution attracts considerable attention. For more detailed descriptions, one can refer to [1-6] and the references therein.

Recently, from the point of view of the system of the linear equations, some efficient numerical methods for solving the large and sparse $\operatorname{LCP}(q, M)$ are developed. Especially, based on the implicit fixed-point equation of the LCP $(q, M)$, a class of modulus iteration method in [7] (see Section 9.2 in [3] as well) and its various versions have been presented in the literature. The goal of modulus iteration method is to take $z=|x|+x$ and $w=|x|-x$ such that the
$\operatorname{LCP}(q, M)$ can be equivalently transformed into a system of fixed-point equations:

$$
(I+M) x=(I-M)|x|-q .
$$

In this way, based on fixed-point equation (2), the modulus iteration method is described as follows.

Modulus Iteration Method. Given an initial vector $x^{(0)} \in \mathbb{R}^{n}$, compute $x^{(k+1)} \in \mathbb{R}^{n}$ by solving the following linear system:

$$
(I+M) x^{(k+1)}=(I-M)\left|x^{(k)}\right|-q .
$$

Then, set

$$
z^{(k+1)}=\left|x^{(k+1)}\right|+x^{(k+1)}, \quad k=0,1,2, \ldots,
$$

until the iteration sequence $\left\{z^{(k)}\right\}_{k=1}^{+\infty} \subset \mathbb{R}^{n}$ is convergent.

Making the simple substitution $\alpha I$ for $I$ in (3) results in the modified modulus iteration method, which was considered in [8]. Numerical results in [8] showed that the modified modulus method is feasible when the involved matrix $M$ is symmetric positive definite. In [9], combining the modulus method with the matrix splitting of the matrix $M$, a class of modulus-based matrix splitting iteration methods is developed, which not only includes some 
presented iteration methods, such as the modified modulus method [8] and nonstationary extrapolated modulus algorithms [10] but also yields a series of iteration methods, such as modulus-based Jacobi, Gauss-Seidel, SOR, and AOR iteration methods. Further discussing the modulus-based matrix splitting iteration method and its various versions, one can see [11-17] for more details. In addition, for other forms of iteration methods, one can see [18-22].

In this paper, we focus on this situation where the involved matrix $M$ of the $\operatorname{LCP}(q, M)$ in (1) is non-Hermitian positive definite. By reformulating equivalently the implicit fixed-point equation of the $\operatorname{LCP}(q, M)$ as a two-by-two block nonlinear equation, based on the GSOR iteration method in [23], we extend the GSOR iteration method for the $\operatorname{LCP}(q, M)$ in (1) with its two-by-two block form. That is to say, we present a generalized SOR-like iteration method to solve the $\operatorname{LCP}(q, M)$. The convergence conditions of the generalized SOR-like iteration method are discussed under suitable choices of the involved parameter. Numerical examples are reported to show that the generalized SOR-like iteration method is feasible and effective in computing.

For our analysis, here we briefly explain some terminologies used in the next section. Let $\mathbb{R}^{n}$ be the finite dimension Euclidean space, whose norm is denoted by $\|\cdot\|$. For $x \in \mathbb{R}^{n}, \operatorname{sign}(x)$ denotes a vector with components equal to $1,0,-1$ depending on whether the corresponding component of $x$ is positive, zero, or negative. The diagonal matrix $D(x)=\operatorname{diag}(\operatorname{sign}(x))$ denotes a diagonal matrix corresponding to $\operatorname{sign}(x)$. Matrix $A$ is called a non-Hermitian positive definite matrix if its Hermitian part, $1 / 2\left(A+A^{T}\right)$, is positive definite.

This paper is organized as follows. In Section 2, the generalized SOR-like iteration method is established and its convergence properties are discussed. In Section 3, the generalized SOR-like iteration method is used to solve the absolute value equation (AVE). Numerical experiments are reported in Section 4, and finally, some concluding remarks are given in Section 5 .

\section{Generalized SOR-Like Iteration Method}

In this section, the generalized SOR-like iteration method is established. To this end, we take $z=|x|+x$ and $w=\Omega(|x|-x)$, where $\Omega$ is a nonnegative diagonal matrix, and then the $\operatorname{LCP}(q, M)$ can be equivalently transformed into the following fixed-point equations:

$$
(\Omega+M) x=(\Omega-M)|x|-q .
$$

Let $y=|x|$. From (5), we obtain

$$
\left\{\begin{array}{l}
(\Omega+M) x-(\Omega-M)|x|=-q, \\
-|x|+y=0
\end{array}\right.
$$

that is,

$$
\bar{A} z=\left(\begin{array}{cc}
\Omega+M & -(\Omega-M) \\
-D(x) & I
\end{array}\right)\left(\begin{array}{l}
x \\
y
\end{array}\right)=\left(\begin{array}{c}
-q \\
0
\end{array}\right)=\bar{b},
$$

where $D(x)=\operatorname{diag}(\operatorname{sign}(x)), x \in \mathbb{R}^{n}$.
Let

$$
\begin{aligned}
& \bar{\Omega}=\left(\begin{array}{cc}
\omega I & 0 \\
0 & \tau I
\end{array}\right), \\
& \bar{A}=\mathscr{L}-\mathscr{L}-\mathscr{U},
\end{aligned}
$$

where

$$
\begin{aligned}
& \mathscr{D}=\left(\begin{array}{cc}
\Omega+M & 0 \\
0 & I
\end{array}\right), \\
& \mathscr{L}=\left(\begin{array}{cc}
0 & 0 \\
D(x) & 0
\end{array}\right), \\
& \mathscr{U}=\left(\begin{array}{cc}
0 & \Omega-M \\
0 & 0
\end{array}\right) .
\end{aligned}
$$

Then, the iteration scheme of the generalized SOR-like iteration method is

$$
\left(\begin{array}{l}
x^{(k+1)} \\
y^{(k+1)}
\end{array}\right)=M_{\omega, \tau}\left(\begin{array}{l}
x^{(k)} \\
y^{(k)}
\end{array}\right)+(\mathscr{D}-\bar{\Omega} \mathscr{L})^{-1} \bar{\Omega}\left(\begin{array}{c}
-q \\
0
\end{array}\right),
$$

where $M_{\omega, \tau}=(\mathscr{D}-\bar{\Omega} \mathscr{L})^{-1}[(I-\bar{\Omega}) \mathscr{D}+\bar{\Omega} \mathscr{U}]$ and $\omega, \tau>0$. Furthermore, the generalized SOR-like method can be described as follows.

The Generalized SOR-Like Iteration Method. Let $M \in \mathbb{R}^{n \times n}$ be a non-Hermitian positive definite matrix, $\Omega$ be a nonnegative diagonal matrix, and $\omega, \tau>0$. Given initial vectors $x^{(0)} \in \mathbb{R}^{m}$ and $y^{(0)} \in \mathbb{R}^{n}$, for $k=0,1,2, \ldots$, until the iteration sequence $\left\{x^{(k)}, y^{(k)}\right\}_{k=0}^{+\infty}$ is convergent, compute

$$
\left\{\begin{array}{l}
x^{(k+1)}=(1-\omega) x^{(k)}+\omega(\Omega+M)^{-1}\left((\Omega-M) y^{(k)}-q\right) \\
y^{(k+1)}=(1-\tau) y^{(k)}+\tau\left|x^{(k+1)}\right|
\end{array}\right.
$$

When $\omega=\tau$ in (11), the generalized SOR-like iteration method reduces to the SOR-like method [24]. That is to say, the generalized SOR-like iteration method is a generalization form of the SOR-like method [24].

Let $\left(x^{*}, y^{*}\right)$ be the solution pair of the equation (7) and $\left(x^{(k)}, y^{(k)}\right)$ be generated by the iteration method (11). Let the iteration errors be

$$
\begin{aligned}
& e_{k}^{x}=x^{*}-x^{(k)}, \\
& e_{k}^{y}=y^{*}-y^{(k)} .
\end{aligned}
$$

Then, we give the following main result with respect to generalized SOR-like iteration method (11).

Theorem 1. Let $M \in \mathbb{R}^{n \times n}$ be non-Hermitian positive definite and

$$
\delta=\left\|(\Omega+M)^{-1}(\Omega-M)\right\| .
$$


If

$$
\begin{aligned}
0 & <\omega, \\
\tau & <2, \\
\omega \tau \delta & <(1-|1-\omega|)(1-|1-\tau|),
\end{aligned}
$$

then

$$
\left\|\left(e_{k+1}^{x}, e_{k+1}^{y}\right)\right\|<\left\|\left(e_{k}^{x}, e_{k}^{y}\right)\right\|, \quad k=0,1, \ldots,
$$

where

$$
\left\|\left(e_{k}^{x}, e_{k}^{y}\right)\right\|=\sqrt{\left\|e_{k}^{x}\right\|^{2}+\left\|e_{k}^{y}\right\|^{2}} .
$$

This implies that the generalized SOR-like method is convergent.

Proof. By the simple computations, from equations (7) and (11), we have

$$
\left\{\begin{array}{l}
e_{k+1}^{x}=(1-\omega) e_{k}^{x}+\omega(\Omega+M)^{-1}(\Omega-M) e_{k}^{y} \\
e_{k+1}^{y}=(1-\tau) e_{k}^{y}+\tau\left(\left|x^{*}\right|-\left|x^{(k+1)}\right|\right) .
\end{array}\right.
$$

From (17), we can obtain

$$
\begin{aligned}
\left\|e_{k+1}^{x}\right\| & \leq|1-\omega| \cdot\left\|e_{k}^{x}\right\|+\omega \delta\left\|e_{k}^{y}\right\|, \\
\left\|e_{k+1}^{y}\right\| & \leq|1-\tau|\left\|e_{k}^{y}\right\|+\tau\left\|\left|x^{*}\right|-\left|x^{(k+1)}\right|\right\| \\
& \leq|1-\tau|\left\|e_{k}^{y}\right\|+\tau\left\|x^{*}-x^{(k+1)}\right\| \\
& =|1-\tau|\left\|e_{k}^{y}\right\|+\tau\left\|e_{k+1}^{x}\right\| .
\end{aligned}
$$

Furthermore,

$$
\begin{aligned}
\left(\begin{array}{c}
\left\|e_{k+1}^{x}\right\| \\
\left\|e_{k+1}^{y}\right\|
\end{array}\right) & \leq\left(\begin{array}{cc}
|1-\omega| & \omega \delta \\
\tau|1-\omega| & |1-\tau|+\omega \tau \delta
\end{array}\right)\left(\begin{array}{l}
\left\|e_{k}^{x}\right\| \\
\left\|e_{k}^{y}\right\|
\end{array}\right) \\
& \leq\left(\begin{array}{cc}
|1-\omega| & \omega \delta \\
\tau|1-\omega| & |1-\tau|+\omega \tau \delta
\end{array}\right)^{2}\left(\begin{array}{l}
\left\|e_{k-1}^{x}\right\| \\
\left\|e_{k-1}^{y}\right\|
\end{array}\right), \\
& \cdots, \\
& \leq\left(\begin{array}{cc}
|1-\omega| & \omega \delta \\
\tau|1-\omega| & |1-\tau|+\omega \tau \delta
\end{array}\right)^{k}\left(\begin{array}{l}
\left\|e_{0}^{x}\right\| \\
\left\|e_{0}^{y}\right\|
\end{array}\right) .
\end{aligned}
$$

Let

$$
\bar{M}=\left(\begin{array}{cc}
|1-\omega| & \omega \delta \\
\tau|1-\omega| & |1-\tau|+\omega \tau \delta
\end{array}\right),
$$

and $\rho(\bar{M})$ denote the spectral radius of matrix $\bar{M}$. When $\rho(\bar{M})<1$, the generalized SOR-like method (11) converges. Let $\lambda$ be an eigenvalue of the matrix $\bar{M}$. Then, $\lambda$ satisfies

$$
(\lambda-|1-\omega|)(\lambda-|1-\tau|-\omega \tau \delta)-\tau \omega \delta|1-\omega|=0,
$$

which is equal to

$$
\lambda^{2}-(|1-\omega|+|1-\tau|+\omega \tau \delta) \lambda+|1-\omega| \cdot|1-\tau|=0 .
$$

Applying Lemma 2.1 in [25] for equation (23), $|\lambda|<1$ is equivalent to

$|1-\omega| \cdot|1-\tau|<1$ and $|1-\omega|+|1-\tau|+\omega \tau \delta<1+|1-\omega| \cdot|1-\tau|$.

Therefore, if condition (14) holds, then $\rho(\bar{M})<1$. This completes the proof.

Clearly, when $\omega=\tau$, then the generalized SOR-like method reduces to the SOR-like method. For the SOR-like method, we have the following corollary.

Corollary 1. Let $M \in \mathbb{R}^{n \times n}$ be non-Hermitian positive definite and

$$
\delta=\left\|(\Omega+M)^{-1}(\Omega-M)\right\| .
$$

If

$$
0<\omega<2 \text { and } \omega^{2} \delta<(1-|1-\omega|)^{2},
$$

then

$$
\left\|\left(e_{k+1}^{x}, e_{k+1}^{y}\right)\left|\|<\|\left(e_{k}^{x}, e_{k}^{y}\right)\right|\right\|, \quad k=0,1, \ldots
$$

where

$$
\left\|\left(e_{k}^{x}, e_{k}^{y}\right)\right\|=\sqrt{\left\|e_{k}^{x}\right\|^{2}+\left\|e_{k}^{y}\right\|^{2}} .
$$

This implies that the SOR-like method is convergent.

\section{Generalized SOR-Like Method for AVE}

On the basis of Proposition 2 in [26], the linear complementarity problem (LCP) and the absolute value equations (AVEs) are equivalent under certain conditions. Based on this, in this section, we will extend the generalized SOR-like method for the following AVE:

$$
A x-|x|=b,
$$

where $A \in \mathbb{R}^{n \times n}, b \in \mathbb{R}^{n}$, and $|x|$ denotes all the components of the vector $x \in \mathbb{R}^{n}$ by absolute value.

Based on the results in Section 2, it is easy to obtain that the generalized SOR-like method for AVE (29) can be established and described as follows.

The Generalized SOR-Like Iteration Method for the $A V E$. Let $A \in \mathbb{R}^{n \times n}$ be nonsingular and $\omega, \tau>0$. Given initial vectors $x^{(0)} \in \mathbb{R}^{m}$ and $y^{(0)} \in \mathbb{R}^{n}$, for $k=0,1,2, \ldots$, until the iteration sequence $\left\{x^{(k)}, y^{(k)}\right\}_{k=0}^{+\infty}$ is convergent, compute

$$
\left\{\begin{array}{l}
x^{(k+1)}=(1-\omega) x^{(k)}+\omega A^{-1}\left(y^{(k)}+b\right), \\
y^{(k+1)}=(1-\tau) y^{(k)}+\tau\left|x^{(k+1)}\right|
\end{array}\right.
$$

It is easy to see that we use $A^{-1}$ instead of $(\Omega+M)^{-1}(\Omega-M)$ in Theorem 1, so the convergence condition of the generalized SOR-like iteration method for AVE (29) is obtained. 
Theorem 2. Let $A \in \mathbb{R}^{n \times n}$ be nonsingular and $\nu=\left\|A^{-1}\right\|$. If

$$
\begin{aligned}
0 & <\omega, \\
\tau & <2, \\
\omega \tau \nu & <(1-|1-\omega|)(1-|1-\tau|),
\end{aligned}
$$

then

$$
\left\|\left(e_{k+1}^{x}, e_{k+1}^{y}\right)\right\|\|\|\left(e_{k}^{x}, e_{k}^{y}\right)\|\|, \quad k=0,1, \ldots
$$

where

$$
\left\|\left(e_{k}^{x}, e_{k}^{y}\right) \mid\right\|=\sqrt{\left\|e_{k}^{x}\right\|^{2}+\left\|e_{k}^{y}\right\|^{2}}
$$

this implies that the generalized SOR-like method is convergent.

Furthermore, for the SOR-like method, we have the following corollary.

Corollary 2. Let $A \in \mathbb{R}^{n \times n}$ be nonsingular and $\nu=\left\|A^{-1}\right\|$. Denote

$$
\begin{aligned}
& \alpha=|1-\omega|, \\
& \beta=\omega^{2} \nu .
\end{aligned}
$$

If

$$
\begin{aligned}
0 & <\omega<2, \\
\alpha+\sqrt{\beta} & <1,
\end{aligned}
$$

then

$$
\left\|\left(e_{k+1}^{x}, e_{k+1}^{y}\right)\right\|<\left\|\left(e_{k}^{x}, e_{k}^{y}\right)\right\| \|, \quad k=0,1, \ldots
$$

where

$$
\left\|\left(e_{k}^{x}, e_{k}^{y}\right) \mid\right\|=\sqrt{\left\|e_{k}^{x}\right\|^{2}+\left\|e_{k}^{y}\right\|^{2}}
$$

This implies that the SOR-like method is convergent.

Comparing Corollary 2 with Theorem 3.1 in [24], it is easy to see that the region of the parameter $w$ in Corollary 2 is the same as that in Theorem 3.1 in [24]. Both require $0<\omega<2$ in Corollary 2 and Theorem 3.1 in [24]. The difference between Corollary 2 and Theorem 3.1 in [24] is on $\alpha$ and $\beta$. The former is $\alpha+\sqrt{\beta}<1$, and the latter is

$$
\alpha^{4}-3 \alpha^{2}-2 \alpha \beta-2 \beta^{2}+1>0
$$

see Theorem 3.1 in [24]. Formally, the former is simpler than the latter.

\section{Numerical Experiments}

In this section, two examples are given to illustrate the feasibility and effectiveness of the generalized SOR-like method in terms of iteration steps (denoted by "IT") and computing time (denoted by "CPU”). Here, all initial vectors are chosen to be

$$
x^{(0)}=(1,0,1,0, \ldots, 1,0, \ldots)^{T} \in \mathbb{R}^{n} .
$$

All iterations are terminated once $\operatorname{RES}\left(z^{(k)}\right) \leq 10^{-6}$, where "RES" is defined as

$$
\operatorname{RES}\left(z^{(k)}\right):=\left\|\min \left(M z^{(k)}+q, z^{(k)}\right)\right\|,
$$

with $z^{(k)}$ being the $k$-th approximate solution to the $\operatorname{LCP}(q, M)$ and the minimum being taken componentwise in [9]. All the tests are performed in MATLAB 7.0.

To show the advantage of the generalized SOR-like method, we compare the numerical results of the generalized SOR-like method with the SOR-like method [24] and the modulus-based SOR method [9].

In our computations, for the sake of convenience, we take $\Omega=12 I$ in the generalized SOR-like method, the SORlike method [24], and the modulus-based SOR method [9].

In the following tables, "GSOR" denotes the generalized SOR-like method, "SOR" denotes the SOR-like method [24], "MSOR" denotes the modulus-based SOR method [9], and “-” denotes the CPU times larger than 500 seconds or the iteration numbers larger than 500 steps.

Example 1 (see [9]). Let $\operatorname{LCP}(q, M)$ in (1) with

$$
\begin{aligned}
M & =\widehat{M}+\mu I_{n} \in \mathbb{R}^{n \times n}, \quad \widehat{M}=\operatorname{tridiag}\left(-I_{m}, S,-I_{m}\right) \in \mathbb{R}^{n \times n}, \\
S & =\operatorname{tridiag}(-1,4,-1) \in \mathbb{R}^{m \times m}, \quad \mu=4,
\end{aligned}
$$

$$
z^{*}=(1,2,1,2, \ldots, 1,2, \ldots)^{T} \in \mathbb{R}^{n}
$$

be the unique solution of the $\operatorname{LCP}(q, M)$.

In Tables 1 and 2, for different problem sizes of $n$, we list the iteration steps, the CPU times with the generalized SORlike method, the SOR-like method, and the modulus-based SOR method. From the numerical results in Tables 1 and 2, we observe that the modulus-based SOR method fails to converge in 500 iterations. The generalized SOR-like method and the SOR-like method converge and quickly compute a satisfactory approximation to the solution of the $\operatorname{LCP}(q, M)$. Furthermore, it is easy to see that the generalized SOR-like method requires less iteration steps than the SOR-like method. Moreover, the generalized SOR-like method costs less CPU times than the SOR-like method. Therefore, in terms of computing efficiency, the generalized SOR-like method outperforms both the SOR-like method and the modulus-based SOR method under certain conditions.

Example 2 (see [9]). Let $\operatorname{LCP}(q, M)$ in (1) with

$$
\begin{aligned}
M & =\widehat{M}+\mu I_{n} \in \mathbb{R}^{n \times n}, \quad \widehat{M}=\operatorname{tridiag}\left(-1.5 I_{m}, S,-0.5 I_{m}\right) \in \mathbb{R}^{n \times n}, \\
S & =\operatorname{tridiag}(-1.5,4,-0.5) \in \mathbb{R}^{m \times m}, \quad \mu=4,
\end{aligned}
$$

$z^{*}=(1,2,1,2, \ldots, 1,2, \ldots)^{T} \in \mathbb{R}^{n}$

be the unique solution of the $\operatorname{LCP}(q, M)$.

In Tables 3 and 4 , for different problem sizes of $n$, we list the iteration steps and the CPU times with respect to the generalized SOR-like method, the SOR-like method, and the modulus-based SOR method. These numerical results 
TABLE 1: CPU and IT for GSOR, SOR, and MSOR with $n=900$ and $\omega=1$.

\begin{tabular}{cccccccc}
\hline & $\tau$ & 1.1 & 1.2 & 1.5 & 1.6 & SOR & MSOR \\
\hline GSOR & IT & 23 & 20 & 18 & 24 & 26 & - \\
& CPU & 0.079 & 0.078 & 0.047 & 0.063 & 0.093 & - \\
\hline
\end{tabular}

TABLE 2: CPU and IT for GSOR, SOR, and MSOR with $n=3600$ and $\omega=1$.

\begin{tabular}{cccccccc}
\hline & $\tau$ & 1.1 & 1.2 & 1.5 & 1.6 & SOR & MSOR \\
\hline GSOR & IT & 24 & 21 & 19 & 24 & 27 & - \\
& CPU & 0.344 & 0.266 & 0.234 & 0.297 & 0.36 & - \\
\hline
\end{tabular}

TABLE 3: CPU and IT for GSOR, SOR, and MSOR with $n=900$ and $\omega=1$.

\begin{tabular}{cccccccc}
\hline & $\tau$ & 1.1 & 1.2 & 1.5 & 1.6 & SOR & MSOR \\
\hline GSOR & IT & 23 & 20 & 19 & 25 & 26 & - \\
& CPU & 0.079 & 0.062 & 0.061 & 0.094 & 0.11 & - \\
\hline
\end{tabular}

TABle 4: CPU and IT for GSOR, SOR, and MSOR with $n=3600$ and $\omega=1$.

\begin{tabular}{cccccccc}
\hline & $\tau$ & 1.1 & 1.2 & 1.5 & 1.6 & SOR & MSOR \\
\hline GSOR & IT & 24 & 21 & 20 & 26 & 27 & - \\
& CPU & 0.343 & 0.281 & 0.279 & 0.36 & 0.391 & - \\
\hline
\end{tabular}

further confirm the observations obtained from Tables 1 and 2, i.e., the generalized SOR-like method is superior to both the SOR-like method and the modulus-based SOR method in terms of computing efficiency under certain conditions.

\section{Conclusion}

In this paper, we have presented a generalized SOR-like iteration method for solving the non-Hermitian positive definite linear complementarity problem (LCP) in (1), which is obtained by reformulating equivalently the implicit fixedpoint equation of the LCP as a two-by-two block nonlinear equation. Some convergence properties of the generalized SOR-like iteration method are obtained. That is, the generalized SOR-like iteration method can converge to the solution of the LCP in (1) under suitable choices of the involved parameter. Numerical experiments have been reported to confirm the efficiency of the proposed method.

\section{Data Availability}

The data used to support the findings of this study are included within the article.

\section{Conflicts of Interest}

The authors declare that there are no conflicts of interest regarding the publication of this paper.

\section{Acknowledgments}

This research was supported by the National Natural Science Foundation of China (No. 11961082).

\section{References}

[1] R. W. Cottle and G. B. Dantzig, "Complementary pivot theory of mathematical programming," Linear Algebra and Its Applications, vol. 1, no. 1, pp. 103-125, 1968.

[2] R. W. Cottle, J.-S. Pang, and R. E. Stone, The Linear Complementarity Problem, Academic, San Diego, CA, USA, 1992.

[3] K. G. Murty, Linear Complementarity, Linear and Nonlinear Programming, Heldermann, Berlin, Germany, 1988.

[4] U. Schäfer, "A linear complementarity problem with a P-matrix," SIAM Review, vol. 46, no. 2, pp. 189-201, 2004.

[5] B. H. Ahn, "Iterative methods for linear complementarity problems with upper-bounds on primary variables," Mathematical Programming, vol. 26, pp. 295-315, 1983.

[6] S.-L. Wu and C.-X. Li, "A generalized Newton method for non-hermitian positive definite linear complementarity problem," Calcolo, vol. 54, no. 1, pp. 43-56, 2017.

[7] W. Van Bokhoven, Piecewise-linear Modelling and Analysis, Proefschrift, Eindhoven, Netherlands, 1981.

[8] J.-L. Dong and M.-Q. Jiang, "A modified modulus method for symmetric positive-definite linear complementarity problems," Numerical Linear Algebra with Applications, vol. 16, no. 2, pp. 129-143, 2009.

[9] Z.-Z. Bai, "Modulus-based matrix splitting iteration methods for linear complementarity problems," Numerical Linear Algebra with Applications, vol. 17, no. 6, pp. 917-933, 2010.

[10] A. Hadjidimos and M. Tzoumas, "Nonstationary extrapolated modulus algorithms for the solution of the linear complementarity problem," Linear Algebra and Its Applications, vol. 431, no. 1-2, pp. 197-210, 2009.

[11] W. Li, "A general modulus-based matrix splitting method for linear complementarity problems ofH-matrices," Applied Mathematics Letters, vol. 26, no. 12, pp. 1159-1164, 2013.

[12] W.-W. Xu and H. Liu, "A modified general modulus-based matrix splitting method for linear complementarity problems of H-matrices," Linear Algebra and Its Applications, vol. 458, pp. 626-637, 2014.

[13] A. Hadjidimos, M. Lapidakis, and M. Tzoumas, "On iterative solution for linear complementarity problem with an $\$ \mathrm{H}_{-}$ $\{+\}$ \$-Matrix," SIAM Journal on Matrix Analysis and Applications, vol. 33, no. 1, pp. 97-110, 2012.

[14] S.-L. Wu and C.-X. Li, "Two-sweep modulus-based matrix splitting iteration methods for linear complementarity problems," Journal of Computational and Applied Mathematics, vol. 302, pp. 327-339, 2016.

[15] L.-L. Zhang and Z.-R. Ren, "Improved convergence theorems of modulus-based matrix splitting iteration methods for linear complementarity problems," Applied Mathematics Letters, vol. 26, no. 6, pp. 638-642, 2013.

[16] N. Zheng and J.-F. Yin, "Accelerated modulus-based matrix splitting iteration methods for linear complementarity problem," Numerical Algorithms, vol. 64, no. 2, pp. 245-262, 2013.

[17] N. Zheng and J.-F. Yin, "Convergence of accelerated modulus-based matrix splitting iteration methods for linear complementarity problem with anH+-matrix," Journal of Computational and Applied Mathematics, vol. 260, pp. 281293, 2014. 
[18] H. S. Najafi and S. A. Edalatpanah, "Modification of iterative methods for solving linear complementarity problems," Engineering Computations, vol. 30, pp. 910-923, 2013.

[19] S. A. Edalatpanah, "SOR-like methods for non-hermitian positive definite linear complementarity problems," AMOAdvanced Modeling and Optimization, vol. 15, no. 3, pp. 697-704, 2013.

[20] X. Mao, X. Wang, S. A. Edalatpanah, and M. Fallah, "The monomial preconditioned SSOR method for linear complementarity problem," IEEE Access, vol. 7, pp. 73649-73655, 2019.

[21] W. Wang, Z. Zhou, S. A. Edalatpanah, and S. E. Najafi, "A new approach for the modulus-based matrix splitting algorithms," IEEE Access, vol. 1, no. 1, p. 99, 2019.

[22] S. A. Edalatpanah, "On the preconditioned projective iterative methods for the linear complementarity problems," RAIRO Operations Research, vol. 54, no. 2, pp. 341-349, 2020.

[23] Z.-Z. Bai, B. N. Parlett, and Z.-Q. Wang, "On generalized successive overrelaxation methods for augmented linear systems," Numerische Mathematik, vol. 102, no. 1, pp. 1-38, 2005.

[24] Y.-F. Ke and C.-F. Ma, "SOR-like iteration method for solving absolute value equations," Applied Mathematics and Computation, vol. 311, pp. 195-202, 2017.

[25] S.-L. Wu, T.-Z. Huang, and X.-L. Zhao, "A modified SSOR iterative method for augmented systems," Journal of Computational and Applied Mathematics, vol. 228, no. 1, pp. 424-433, 2009.

[26] O. L. Mangasarian and R. R. Meyer, "Absolute value equations," Linear Algebra and Its Applications, vol. 419, no. 2-3, pp. 359-367, 2006. 